\title{
A Situated Approach to Systems Based Modeling of Services
}

\author{
Anshuman Saxena ${ }^{1,2}$ \\ ${ }^{1}$ Systemic Modeling Laboratory, I\&C, EPFL Switzerland \\ ${ }^{2}$ TCS Innovation Labs, Bangalore India \\ anshuman.saxena@\{epfl.ch, tcs.com\}
}

\author{
Alain Wegmann \\ Systemic Modeling Laboratory (LAMS) \\ I\&C, EPF-Lausanne, CH 1015 Switzerland \\ alain.wegmann@epfl.ch
}

\begin{abstract}
Situatedness refers to the imagery that a conceptualization invokes. The image, as a whole, provides the context for interpreting the relevance of the categories revealed in the image. At a basic level of conceptualization, the conceptual relevance of an observed category can be historically inspired, empirically informed, rationally thought, or pragmatically focused. From a service-design perspective, the interest in a given phenomenon is limited to its functional organization, which requires taking an exclusively pragmatic view of the world. In this paper, we propose a role-based approach to modeling categories, which requires service-designers assign functional and non-functional roles to categories by making explicit their interpretation of the conceptual relevance of these categories. Staying aware of the design choices will help the service-designer develop an informed model of observed reality, leading to better alignment between the scope and the purpose of the inquiry.
\end{abstract}

Keywords-causation; force-dynamics; functional roles; situated refinement; public policy

\section{INTRODUCTION}

Systems is a theory of observation [1]. Observations are appreciative judgments that reveal the categories observed in a phenomenon of interest [2]. A Systems approach to inquiry helps the modeler organize these categories into groups such that the behavior exhibited by the interaction between these categories is preserved [3]. In the context of service-oriented inquiry, the service-designer is interested in specifying the behavior required to create value for some particular individual or group of individuals. Value is experiential in nature. In a world rich in variety, to ensure that some target subject undergoes a certain experience, it is important that the design of the behavior intended to deliver that experience, internalizes, as much as possible, the real world uncertainties that may influence this behavior [4]. As a result, Systems based approach to services, adopts a dichotomous approach to categorization. For a given phenomenon of interest, it first identifies the category corresponding to the target subject, henceforth referred to as the Focal-element. All other categories identified as relevant to the phenomenon of interest are grouped together into one system, commonly referred to as the Service-system [5]. The independent standing of the Focalelement is to emphasize the subjectivity of the experience that the behavior of the Service-system creates for the target subject. On the other hand, Service-system conveys the notion of functional unity - everything that can influence, either intentionally or un-intentionally, the creation of the intended experience for the Focal-element is seen as functionally-joined in their interaction with the Focal-element. Figure 1 provides a visual depiction of the service as the behavior exhibited by the Service-system and the experience it creates for the Focalelement.

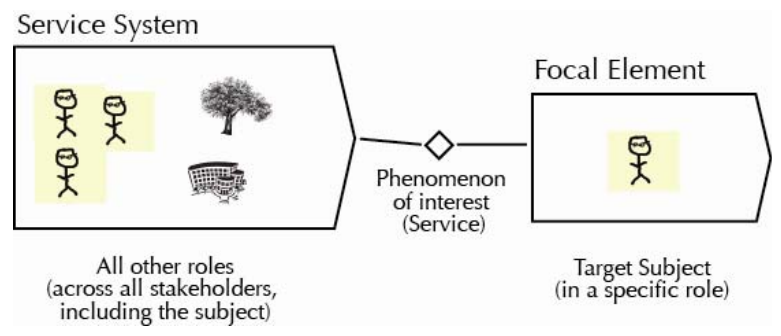

Figure 1. Service-oriented systems model of phenomenon of interest

Existing approach to service modeling [6], reify systems through a stakeholder mapping process. Stakeholders are entities or group of entities that are active in one or more causeand-effect relationships constituting the phenomenon of interest. The Service-system is mapped to the set of entities, which design, deliver and operate the service. The Focalelement is mapped to an entity, either an individual or a group of individuals, which consumes the service. Such an entitybased instantiation of systems is ontological in nature and may not always reflect the real semantics of the phenomenon of interest. An entity may participate in more than one aspect of the phenomenon of interest and the nature of participation may vary across different situations. To ensure that a Systems model is a meaningful representation of the observed reality, it is important that we capture the deeper aspects of an entities' participation to the phenomenon of interest. 
Roles is a semantic category that can be used to re-define stakeholders by contextualizing their participation in a given phenomenon of interest. Roles reveal the semantics of an entity's participation to a situation. Nevertheless, roles should not be seen as truth-conditional labels [7]. Roles are grounded in the modeler's conceptualization of the observed reality. Conceptualization is imagistic in nature [8] and is enabled through different epistemic considerations such as historicism, empiricism, rationalism and pragmatism [9]. It is the contribution of these different epistemological views in activating an imagined situation that lends semantics its encyclopedic nature [10]. As a result, at a basic-level of conceptualization [11], an entity's participation to a situation may not necessarily reveal the entity's role in some cause-andeffect relationship relevant to the phenomenon of interest. Roles may also correspond to an entity's participation to nonfunctional aspects of imagery like cognitive reference roles [12] and foreground/background roles [13]. Since the design of a service is an engineering activity [14], a service designer is inclined to specifically model only those roles undertaken by entities, whose causal linkage to the phenomenon of interest has been exposed.

For example, consider a bike manufacturer conducting an inquiry on use of bike to school. One popular image of a person biking to school is where she carries a bag. The inclusion of bag in the 'biking to school' conceptualization may be grounded in historicism, as historically we have been exposed to the act of carrying reading, writing instruments and lunch box to school. From a bike manufacturer perspective, the role of 'bag-carrier' undertaken by the person biking to school is not, by default, relevant. This, initially historicism inspired, role gains significance for the inquiry only when it is shown to have some causal relevance to biking to school say, increased tiredness due to weight on shoulder, reduced maneuverability in traffic, ergonomic considerations, etc. Figure 2 highlights the different epistemic considerations that contribute to the popular imagery of an individual biking to school.

To further reveal the causal organization of the phenomenon of interest beyond what is, by default, explicit at the basic-level of conceptualization, the service designer needs to further situate its model of observed reality by admitting refinements [15] of basic-level categories. Mapping nonfunctional roles to functional roles can be aided by exploring the initially imagined situation, corresponding to the basic-level conceptualization, at finer levels of granularity. Exploring the situation at finer details of activity generates additional information. Given the computational boundedness of the modeler, increasing the resolution of a situation results in reducing the scope of the situation [16] thereby, keeping the total amount of information to be processed within the modelers computational limits.

In this paper, we reflect on two foundational aspects relevant to Systems based modeling of services. First, we clarify the traditional notion of causation that considers only those situations as causative where an observable change in state occurs. The reference to the differential of states though widely followed, is limited in interpreting causation as only those interactions between entities where one entity inflicts some change in state at the other entity. This does not include

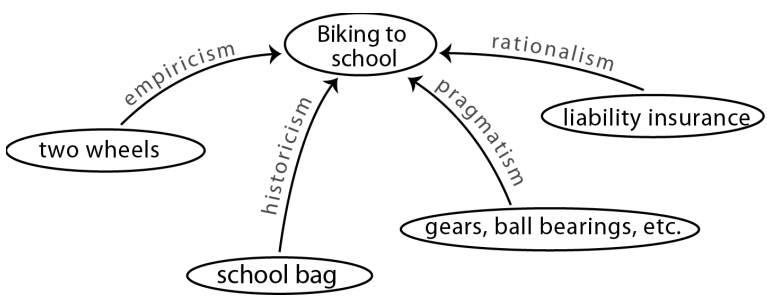

Figure 2. The imagistic nature of conceptualization

situations corresponding to natural language expressions like let and hindrance. From a service design perspective, it is important to include let and hindrance as instances of causeand-effect relationships. As mentioned earlier, services aim at creating value by delivering an experience enriching performance. Value is the change an individual experiences. Nevertheless, this change may not always be in reference to some prior state, it can also be in reference to some anticipated state. For example, contrast the two situations: switching on an air conditioner makes a hot room cold and letting the air conditioner run, even after the room has cooled down, allows it to stay cold. In the first case, it is quite evident that the air conditioner causes the change in state, from a prior hot-room condition to a resultant cold-room condition. In the second case, the change in state is not easily evident as the room continues to be cold. But when viewed in reference to the default situation of the room going back to being hot in summers, it is evident that there is a continuous effort that is being put through the air conditioner to cause the room to stay cold. The change in state here is evident when seen in the context of the default/anticipated state. As for situations corresponding to hindrance, there is no state change since by definition hindrance suggests a weaker force that is not capable of state change. Nevertheless from a service design perspective, knowledge of hindrance still exposes a potential source for state change, which could gain strength with the passage of time and cause noticeable change. To ensure sustainable service design, it is important that we do not limit the notion of functional roles to only those participations of entities, which are associated with explicit state change. Functional roles should be assigned based on a deeper view of causation, which considers a situation causative if there is evidence that entities are exerting mutually opposing forces [17].

Second, we reflect on the refinement process that a service designer undertakes to map the non-functional aspects observed at some higher level of abstraction to functional-roles that may be revealed at lower levels of abstraction. These refinements are a way to specialize the higher-level context by revealing higher resolution situations, which, though limited in their scope, provider greater detail about the entity's role in an activity. Nevertheless, for the inquiry to stay relevant, it is important that the refinement process is tractable. In the context of service-oriented inquiry, an important terminating condition for the refinement process is that the refined situation should be relevant to the purpose of the inquiry. Such a refinement approach situates the inquiry firmly within the scope of the phenomenon of interest, henceforth referred as situated 
refinement. Situated refinement makes the service designer's choice - in terms of her selection of non-functional roles to be investigated further, explicit. Staying aware of the design choices a service designer makes, helps her develop an informed model of observed reality, which eventually leads to better alignment between the scope and the purpose of the inquiry.

The remaining part of the paper is divided into five parts. Section II discusses the imagistic nature of conceptualization and the different epistemologies that contribute to this image formation. It also discusses how this imagistic character of conceptualization is at the heart of the argument that semantics is encyclopedic in nature. Section III dwells on the relevance of Systems for service-oriented modeling and how the notion of service-system can be better activated by adopting an extended view of causation and a situated approach to functional mapping of stakeholders. Section IV presents a case study on the food-grain storage and distribution activity in India. This case study is an outcome of several workshops that were conducted with various government and non-government agencies in India. We present two instances of service-system that emerged from the discussions between workshop participants. These service-systems are shared as examples of real world scenarios where an entity might undertake multiple functional roles, each situated in a different context of the phenomenon of interest. The paper ends with some concluding remarks and future research directions in Section V.

\section{The IMAGistic NATURE OF CONCEPTUALIZATION}

Cognitively, conceptualization is situated [18]. Situatedness refers to the imagery that a conceptualization invokes. It is the reenactment of a combination of prior experiences that together simulate a perceptual experience in the form of a situation experienced or imaginary. The imagistic nature of conceptualization is enabled through different epistemic considerations. The four prominent epistemological theories that are considered relevant from an information science perspective include historicism, empiricism, rationalism and pragmatism. We refer to [9] in introducing these epistemologies:

\section{A. Empiricism}

Empiricism is that epistemic influence on conceptualization, where judgment is based solely on observations. It is considered a bottom-up approach where knowledge is created by virtue of the observer being exposed to a certain sample set and patterns of association formed based on repeated occurrence of a category. In the absence of any theoretical base, the associations are purely symbolic in nature. For example, the popular image of bike, depicted in Figure 2, models bike as having two wheels. Historically, we are in the know of bikes with one wheel. Also, rationally speaking there is no logical reason for only having two wheels in the bike. Pragmatically too, the need to stay put with twowheel bike configuration is not a necessary one - be it in terms of balancing or maneuverability. Nevertheless, our exposure to bikes with two wheels has been considerably higher amongst our overall experience of the bike as a concept. Such numberof-exposure based reinforcement of a concept is referred to as grounded in empiricist consideration.

\section{B. Rationalism}

Rationalism is that epistemic influence on conceptualization, which is grounded in the tradition of logic. Rationally inspired conceptualization exposes the mental model of the modeler who builds the logical argument to deduce a specific inference. For example, the inclusion of liability insurance as an element of the popular image of bike, depicted in Figure 2, reveals the authors' mental model of biking in a country like Switzerland where all risks are appropriated.

\section{Historicism}

Historicism is that epistemic influence on conceptualization, which is based on social contexts and historical developments. It relies on culturally grounded experience that is accumulated overtime. For example, in Figure 2, the inclusion of bag in the 'biking to school' conceptualization may be grounded in historicism, as historically we have been exposed to the act of carrying reading, writing instruments and lunch box to school. This may not necessarily hold today as adoption of IT based teaching methods have made stationary redundant and homepacked lunch has made way for cafeteria food.

\section{Pragmatism}

Pragmatism is that epistemic consideration which is driven by the purpose of the conceptualization. It promotes a functional view of reality where categories are acknowledged by virtue of their relevance to explaining the causal organization of the phenomenon of interest. For example, in Figure 2, the inclusion of ball bearings and gear as part of popular imagery of the bike as a means of transport, exhibits the bike manufacturers' purpose of conducting the inquiry - to come up with new bike designs.

It is the contribution of these different epistemological views in activating an imagined situation that lends semantics its encyclopedic nature. As a result, the categories revealed in a conceptualization may not always undertake a functional role. They may also undertake non-functional roles that contribute to the imagery at the structural level [19]. For example, the mere acknowledgment of the category 'schoolbag' as part of the 'bike to school' conceptualization does not suggest that 'school-bag' has some causal relevance to the phenomenon of biking to school. At this level of conceptualization, 'school-bag' undertakes a more structural role, helping construct an image that has conceptual relevance for the modeler. May be in this case, what distinguishes the image of a 'general bike ride' from the 'bike to school' phenomenon is the inclusion of school bag in the popular imagery of an individual biking to school. Base/profile is a typical non-functional role that interests the image processing community. At the level of conceptualization depicted in Figure 2, the 'school bag' undertakes the role of a cognitive reference point, which helps to profile a category, 'bike to 
school', by referring to a cognitively related category 'school bag'. This is not to suggest that 'school bag' does not have any functional relevance to 'biking to school' phenomenon. Its relevance to the causal organization is revealed when the scope of the 'bike to school' activity is refined to the 'ride to school'. The 'ride to school' has a reduced scope in terms of focusing only on the riding experience of the rider but has a higher resolution in terms of revealing categories like tiredness, maneuverability in traffic etc. It is in this context that 'school bag' undertakes a functional role, as it is part of the cause-effect relation whose relevance to the refined scope of the phenomenon of interest is easily demonstrable.

\section{SERVICE-ORIENTED SYSTEMS MODELING}

Systems is a set of interacting categories, which together exhibit some behavior for an observer, and that this behavior cannot be construed from any subset of these categories [20]. In the context of service-oriented inquiry, a Systems based approach to modeling the phenomenon of interest helps the service-designer in organizing the categories revealed in her conceptualization of observed reality such that it preserves the behavior exhibited by the phenomenon of interest. Nevertheless, given the imagistic nature of conceptualization and the different epistemologies that lend the conceptualization an encyclopedic semantics, the observed behavior can have both functional and non-functional appeal for the modeler. Since the design of a service is an engineering activity, a service designer is inclined to specifically model only those aspects of observed behavior whose causal linkage to the phenomenon of interest has been exposed. As a result, to fully realize the potential of Systems approach to service-oriented modeling, it is important to disambiguate the process of identifying Service-systems, which have functional appeal, from amongst systems with general behavioral appeal. In the following subsections, we clarify two foundational aspects in modeling Service-systems. First, we clarify the notion of causation that is at the heart of understanding the distinction between functional and non-functional participation. Second, we reflect on how non-functional roles can be used to uncover additional causal linkages by situating them in conceptualizations of varied scope and resolution.

\section{A. Force-dynamic interpretation of causation}

Force-dynamics is the study of interaction between entities with respect to the forces that they exert and experience. The force factors included are not limited to the basic usage of force in the physical realm but also other epistemic usages in the psychological and sociological domains, for example, beliefs, norms and other action concepts. It is a notional system that organizes a conceptualization in a way that reveals the causeand-effect relationships underlying the phenomenon of interest. Traditional approach to causation refers to only those interactions between entities where the entity being focused experiences a change in state.

Force-dynamics takes a deeper view of causation that goes beyond the change-in-state constraint. It considers a situation causative if there is evidence that entities are exerting mutually opposing forces. It is not necessary that the resultant force bring about some change in the state of the focal entity. Thus force-dynamics also includes natural language expressions like 'letting' and 'hindrance' as representing causative situations. 'Hindrance' signifies a situation where the target entity experiences some opposition from other entities but is strong enough to resist any change in its state. On the other hand, 'letting' signifies a situation where the target entity, initially blocked by stronger opposing forces exerted by other entities, is later allowed to pursuit its original goal by virtue of the cessation of the opposing force. This highlights another aspect of force-dynamic approach that it is not limited to steady state interpretation of situations but also takes into account the process of interaction, which may include multiple state transitions before a certain steady state is achieved.

\section{B. Situated Refinement}

Service-system conveys the functionally joined nature of a group of entities that influence, intentionally or unintentionally, the creation of an intended experience for some target subject, also referred to as the Focal System. Nevertheless, servicesystem is an abstract concept. Its effectiveness in providing a meaningful model of observed reality, which can help servicedesigners draw useful specifications for engineering the service, relies on it correct instantiation. As discussed earlier, service-systems should not be reified at the entity level. Entities may play several roles and roles may not always be restricted to functional aspects, they may also correspond to an entity's participation to non-functional aspects of imagery like cognitive reference roles and foreground/background roles. Since the design of a service is an engineering activity, a service designer is inclined to specifically model only those roles undertaken by entities, whose causal linkage to the phenomenon of interest has been exposed. Nevertheless, at the basic-level the conceptualization may not reveal enough functional roles to capture the richness of the phenomenon of interest. It, therefore, becomes important to be able to further investigate the non-functional roles revealed at one level of abstraction by refining the conceptualization to additional levels of abstraction. To recall, refinement is the process of identifying mappings between different levels of abstraction [15].

Given that conceptualization is situated [21], refinement of conceptualization is essentially an exercise in altering the scope of the situation that the conceptualization invokes. Theoretically, any role can be mapped to a functional role by changing the scope of the underlying situation. Nevertheless, refinement adds to the semantic distance between the original situation and the resulting situation. Constraint-free refinements, though eventually, may reveal a lower-level functional role corresponding to some higher-level nonfunctional role, the semantic gap between the functional role revealed and the phenomenon of interest may be so large that it may render the entire refinement chain conceptually intractable; for example, the scope degenerating to sub-atomic levels of matter, etc. 


\section{AN ILLUSTRATION OF SERVICE-SYSTEM FROM THE PUBLIC POLICY DOMAIN}

The case study described here pertains to issues of foodgrain storage and distribution in India. This study is an outcome of several workshops that were conducted with various government and non-government organizations in India. Food-grain storage is currently a hotly debated publicpolicy issue in India [22]. India is self-sufficient in food production; it produces enough food grain to feed its 1.2 billion population comfortably and still have surplus to sell internationally. Nevertheless, inefficiencies in food-grain storage and distribution have resulted in almost 200 million food-insecure people in India. Two important government programs are relevant in ensuring the food security of the citizens - FCI and PDS. FCI is the agency, which procures food-grains from farmers and stores it in warehouses for distribution. PDS is the agency, which receives the food-grains from the FCI warehouses and sells them at subsidized price to the common man. FCI follows a centralized storage policy, storing food-grains at select strategic locations across the country. As a result, FCI warehouses often fall short on infrastructure required to store such huge volumes of foodgrains. Further, their far-off location results in significant transportation costs, both while procurement and distribution. To overcome these shortcomings in food-grain storage and distribution, various alternative policies are being discussed. One such policy prescription promotes the idea of rural warehouse scheme [23], [24]. The basic idea here is to adopt a distributed storage approach for food-grains. And use the funds to build these new warehouses from another government program, NREGA [25], that guarantees 100 days of gainful rural employment every year - primarily, unskilled construction jobs. The primary purpose of the workshop was to help develop a better understanding of the food-grain storage and distribution landscape in India and reason about the different aspects of the rural warehouse scheme and its financing through the rural employment guarantee program.

As shown in Figure 3, we started out by listing the primary activities represent the situations that invoked when the participants of the workshop thought about the phenomenon of interest - "Storage and distribution of food grains through convergence of FCI, PDS and NREGA". For ease of illustration purposes, we restrict ourselves to two activities distribution of food-grains and employment for villagers. For both these activities, which represent a refined scope of the phenomenon of interest, modeling the service systems reveals the plurality and diversity of roles that an entity might undertake. Figure 4, shows the service-system for situation corresponding to distribution of food-grains (a), and the service-system for situation corresponding to employment for villagers (b). It is worth noting that the service-systems are reified with participating entities in their functional-roles and that as an entity, The Villager, is seen in multiple functionalroles - as a consumer of food-grains, as a construction worker for the construction of rural warehouse, as a farmer who produces food-grains in the first place, and also as a community member, which provides him the identity (say, below poverty line status) required to be eligible for publicly

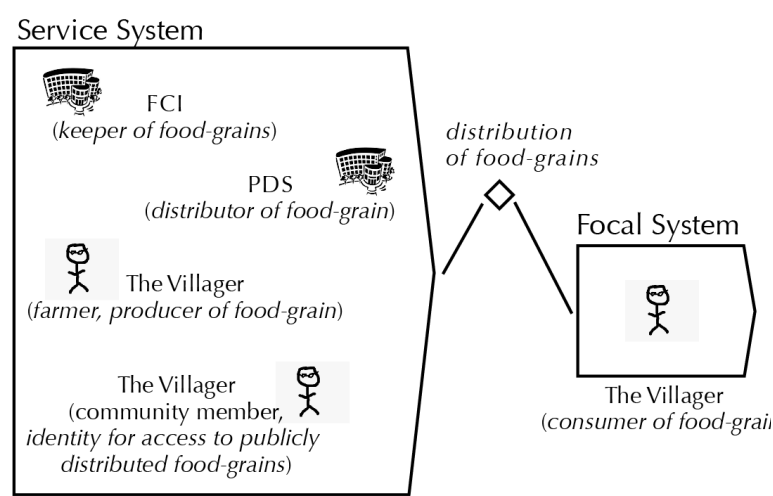

(a)

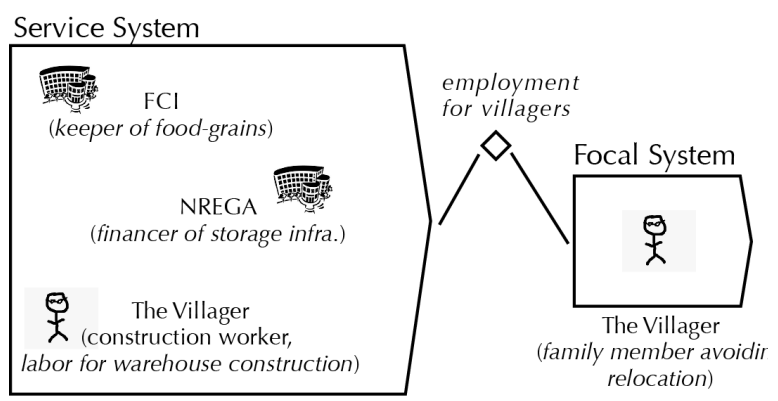

(b)

Figure 4. Example service-systems for the case study

distributed food-grains. An important outcome of the workshop was that the participants of the workshop were able to disambiguate the notion of 'villager', which was, otherwise, used differently in different situations, yet this overloading of the reference to villager was not explicit leading to confusion and conflict of opinions amongst the workshop participants.

\section{CONCLUSIONS AND FUTURE WORK}

Situatedness refers to the imagery that a conceptualization invokes. The image, as a whole, provides the context for interpreting the relevance of the categories revealed in the image. At a basic level of conceptualization, the functional relevance of the observed categories may not, always, be obvious. Categories may be seen as contributing to the image from different perspectives - historically inspired, empirically informed, rationally thought, pragmatically focused. Depending on the epistemological consideration that underlies the category at the basic level, the category may be assigned a functional or non-functional role. Functional roles explicate the causal link of the category to the phenomenon of interest. Nonfunctional roles are limited to the structural aspect of the imagery, which gives the invoked image a unified structure. A category's non-functional contribution to an image, at a given level of abstraction, can be further situated in higher-resolution, reduced-scope imagery at lower levels of abstraction. To ensure that such recursive refinement of imagery does not become intractable, we propose that the service-designer makes the refinement process explicit by clearly articulating how the 
refined scope is relevant to the purpose of the inquiry. Doing so will help develop a service-oriented model of observed reality that is rich in its portrayal of deeper aspects of the phenomenon of interest, while staying firmly focused on the purpose of the inquiry.

An important future research direction for this work is to formalize the conceptual model presented in this paper. We intend to explore a visual semantics oriented formalization of the situated refinement process. From a service design perspective, an obvious next step is to extend this approach to include formalizations of value concept. It is the emphasis on value creation in a world rich in variety that distinguishes the field of Service Science from traditional studies of economic exchange.

\section{REFERENCES}

[1] B. H. Banathy, "Systems inquiry in education," Systemic Practice and Action Research, vol. 1, pp. 193-212, 1988.

[2] D. Schon and G. Wiggins, "Kinds of seeing and their functions in designing," Design Studies, vol. 13, pp. 135-156, 1992.

[3] H. A. Simon, "The Architecture of Complexity," Proceedings of the American Philosophical Society, vol. 106, pp. 467-482, 1962.

[4] S. Alter, "Service system fundamentals: Work system, value chain, and life cycle," IBM Systems Journal, vol. 47, pp. 71-85, 2008.

[5] W. R. Ashby, Introduction to Cybernetics: Methuen, 1964.

[6] H. Chesbrough and J. Spohrer, "A research manifesto for services science," Commun. ACM, vol. 49, pp. 35-40, 2006.

[7] A. Tarski, "The semantic conception of truth: And the foundations of semantics," Philosophy and Phenomenological Research, vol. 4, pp. 341-376, 1944.

[8] L. Barsalou, "Grounded Cognition," Annual Review of Psychology, vol. 59, 2008.

[9] B. Hjørland, "Concept theory," Journal of the American Society for Information Science and Technology, vol. 60, pp. 1519-1536, 2009.

[10] W. Croft and D. A. Cruse, Cognitive Linguistics. Cambridge, UK: Cambridge University Press, 2004.

[11] E. Rosch, "Principles of Categorization," in Cognition and Categorization, E. Roach and B. B. Lloyd, Eds., ed Hillsdale, NJ: Lawrence Erlbaum, 1978, pp. 27-48.

[12] E. Rosch, "Cognitive reference points," Cognitive Psychology, vol. 7, pp. 532-547, 1975.

[13] R. Langacker, Foundations of Cognitive Grammar. Vol. 1: Theoretical Prerequisites. Stanford: Stanford University Press, 1987.

[14] A. B. Saxena and A. Wegmann, "From Composites to Service Systems: The Role of Emergence in Service Design," presented at the IEEE International Conference on Systems, Man, and Cybernetics, Seoul, Korea, 2012.

[15] M. L. Abadi, Leslie, "The existence of refinement mappings," Theor. Comput. Sci., vol. 82, pp. 253-284, 1991.

[16] A. J. Ryan, "Emergence is coupled to scope, not level: Research Articles," Complex., vol. 13, pp. 67-77, 2007.

[17] L. Talmy, "Force Dynamics in Language and Cognition," Cognitive Science, vol. 12, pp. 49-100, 1988.

[18] L. W. Barsalou, "Situated simulation in the human conceptual system," Language and Cognitive Processes, vol. 18, pp. 513-562, 2003.

[19] K. Koffa, Principles of Gestalt Psychology. London: Routledge \& Kegan Paul Ltd, 1935.

[20] M. A. Bedau, "Weak Emergence," Philosophical Perspectives: Mind, Causation, and World, vol. 11, pp. 375-399, 1997.

[21] L. Barsalou and W. Yeh, "The Situated Nature of Concepts," American Journal of Psychology, vol. 119, pp. 349-384, 2006.

[22] B. Mukherji and R. Roy, "India's Grain Storage Comes Up Short: Limited Capacity Threatens Food Supply for Millions of Poor in Second Year in a Row of Bumper Crops," in The Wall Street Journal, ed, 2012.
[23] PIB. (2013). Procurement of Foodgrains For PDS

[24] GOI, "Balancing food-grain demand and supply," Crops Division, Ministry of Agriculture, Government of India, New Delhi2012.

[25] GOI. (2005). National Rural Employment Guarantee Act 2005. 